\title{
Mutagenesis of Phytophthora infestans for Resistance Against Carboxylic Acid Amide and Phenylamide Fungicides
}

Avia (Evgenia) Rubin and Dror Gotlieb, The Mina \& Everard Goodman Faculty of life Sciences, Bar-Ilan University, Ramat-Gan, Israel 52900; Ulrich Gisi, Syngenta Crop Protection, Stein, CH-4332, Switzerland; and Yigal Cohen, The Mina \& Everard Goodman Faculty of life Sciences, Bar-Ilan University, Ramat-Gan, Israel 52900

\begin{abstract}
Rubin, A. E., Gotlieb, D., Gisi, U., and Cohen, Y. 2008. Mutagenesis of Phytophthora infestans for resistance against carboxylic acid amide and phenylamide fungicides. Plant Dis. 92:675-683.

The carboxylic acid amide (CAA) fungicides mandipropamid, dimethomorph, iprovalicarb, and the phenylamide fungicide mefenoxam (MFX, the active enantiomer of metalaxyl) are antioomycete fungicides effective against downy mildews and late blight. Resistance against MFX was reported in nature in several oomycetes including Phytophthora infestans and Plasmopara viticola, whereas resistance against CAAs was reported in $P$. viticola but not in $P$. infestans. In this study the mutability of $P$. infestans for resistance against CAAs and MFX (as a control) was explored under laboratory conditions. UV light or chemical mutagens (e.g., ethyl methan sulfonate [EMS]) were applied to sporangia, and the emergence of mutants resistant to CAAs or MFX, or with altered mating type, was followed. Many mutants resistant to CAAs developed at generation 0 after mutagenesis, but all showed erratic, instable resistance in planta, diminishing after 1 to 8 asexual infection cycles, and failed to grow on CAA-amended medium. In contrast, 19 mutants resistant to MFX were obtained: 6 with UV irradiation (in isolates 28 or 96) and 13 with EMS (in isolates 408, 409, and 410). In three experiments, a shift in mating type, from A1 to A2, was detected. To elucidate whether or not resistance to CAAs is recessive and therefore might emerge only after sexual recombination, A1 and A2 mutants were crossed and the F1 and F2 progeny isolates were tested for resistance. Offspring isolates segregated for resistance to MFX, with resistant isolates maintaining stable resistance in vitro and in planta, whereas all progeny isolates failed to show stable resistance to CAAs in planta or in vitro. The data suggest that $P$. infestans could be artificially mutated for resistance against MFX, but not against CAAs.
\end{abstract}

Additional keywords: cinnamic acid amides, fungicide resistance, mandelic acid amides, mutation, potato, tomato, valinamid carbamates

Mandipropamid (MPD) is a new mandelic acid amide fungicide highly active against foliar oomycete pathogens including Phytophthora infestans and Plasmopara viticola $(5,6,9,11-13,18,19)$. In $P$. viticola, cross resistance was observed between MPD and the valinamide fungicides iprovalicarb (IPRO) and benthiavalicarb and the cinnamic acid amide fungicides dimethomorph (DMM) and flumorph (9). All five compounds were therefore classified as carboxylic acid amide (CAA) fungicides. CAAs share a similar mechanism of fungicidal activity; they specifically inhibit sporangial and cystospore germination (5). The mode of action of CAA fungicides is not known. Some studies $(1,4,13,15,16,19,21,22)$ implicated inhibition of cell wall deposition/assembly, whereas another (10) suggested alterations in phospholipids biosynthesis with an

Corresponding author: Yigal Cohen

E-mail: ycohen@mail.biu.ac.il

Accepted for publication 12 January 2008.

doi:10.1094/PDIS-92-5-0675

(C) 2008 The American Phytopathological Society inhibition of phosphatidylcholine (lecithin) biosynthesis as a main target. Recently, Zhu et al. (28) compared a flumorphsensitive and a flumorph-resistant Phythophthora melonis and suggested that the primary site of action of flumorph is the disruption of F-actin organization.

Sensitivity to MPD was measured in isolates of $P$. infestans collected between 2001 and 2006 from MPD-untreated and -treated fields in Israel and Europe. All isolates were sensitive to MPD (5). Field experiments conducted during 2001 to 2006 showed that selection pressure imposed by repeated sprays of sublethal or excessive doses of MPD on potato or tomato crops infected with mixed field isolates of $P$. infestans produced no resistant isolates against the compound (6). Similarly, repeated sprays with excessive doses of DMM of potato or tomato crops infected with mixed field isolates of $P$. infestans produced no resistant isolates against DMM (24). These results indicated that the probability of buildup of resistant subpopulations of $P$. infestans to CAAs in the field was low.

The lack of resistance to CAAs in $P$. infestans in nature encouraged researchers to produce artificial mutants by applying mainly UV irradiation to mycelia in vitro (26) or $N$-nitroso- $N$-methylurea (NMU) to cystospores (2). Stein and Kirk (26) reported on the generation of dimethomorph resistance by using ethidium bromide/UV light mutagenesis and repeated culturing on DMM-amended medium. After repeated culturing, resistance was increased eightfold or less. DMM resistance resulted in reduced growth rates on nonamended medium and reduced frequency of infection of leaf disks and whole tubers. They concluded that the likelihood for resistance to DMM to appear in nature is negligible. Yuan et al. (27) evaluated the risk of $P$. infestans to develop resistance to flumorph. Mutants resistant to flumorph or metalaxyl were obtained by UV irradiation of mycelia. Metalaxyl-resistant mutants were obtained with a high frequency and exhibited resistance factor values of more than 100, whereas flumorph-resistant mutants were obtained at much lower frequencies and had resistance factors of only 1.5 to $3 \cdot 2$. There was cross-resistance between flumorph and DMM. Most flumorph-resistant mutants showed decrease in hyphal growth in vitro and reduced sporulation both in vitro and on detached leaf tissues. Yuan et al. (27) concluded that the risk of resistance developing was much lower for flumorph than for metalaxyl. Bagirova et al. (2) obtained resistant mutants (resistance factors of only 4 to 7 ) after two-step mutagenesis with NMU. These mutants were less fit in vitro and in planta and reverted back to sensitivity. Most F1 hybrids between the sensitive wild type and the resistant mutants were sensitive. Polygenic, additive inheritance was suggested (2). Recently, Ziogas et al. (29) reported on multi-drug resistance in $P$. infestans that developed in sporangia irradiated with UV $254 \mathrm{~nm}$ for $1 \mathrm{~min}$. The mutants were highly resistant to the CAA fungicides iprovalicarb, benthiavalicarb, and dimethomorph, and several chemically unrelated fungicidal classes including cyazofamid, metalaxyl, cymoxanil, benzamide zoxamide, and chlorothalonil. Pathogenicity tests on wounded stems of tomato seedlings showed that most resistant mutants were significantly less pathogenic compared to the wild-type parent isolate.

The aim of this study was to estimate the likelihood of $P$. infestans developing resistance to the new CAA fungicide MPD as compared to the likelihood of develop- 
ing resistance to the phenylamide fungicide mefenoxam (MFX). This was done by exposing sporangia to UV light or chemical mutagenic agents and examining their capability to infect CAA or MFX-treated leaves. The information provided here is of critical importance, not only for estimating the likelihood of resistance evolution and formulating the resistance management strategy to be employed in the field, but also for studying the biochemical mode of action of CAAs. Preliminary reports on these data were published recently $(24,25)$.

\section{MATERIALS AND METHODS}

Fungal isolates. The following wildtype field isolates of $P$. infestans were used: from the Syngenta collection, 96 (A1, sensitive to MFX) and 28 (A2, sensitive to MFX). From the Bar-Ilan University (BIU) collection, AD (A1, sensitive to MFX), 367 (A2, resistant to MFX) (3), 408, 409, 410, 411, and 412 (all A1, sensitive to MFX). Isolates 96 and 28 were grown on RDA (150 rye seed extract, $10 \mathrm{~g}$ D-glucose, and $20 \mathrm{~g}$ agar in 1 liter of double-distilled water [DDW]) in 9-cm petri dishes at $20^{\circ} \mathrm{C}$, whereas Israeli isolates were maintained by repeated transfer inoculation on detached tomato leaves in petri dishes at $12^{\circ} \mathrm{C}$ and $12 \mathrm{~h}$ light/day.

Chemicals. EMS (ethyl methan sulfonate), EB (ethidium bromide), MNNG (methyl- $N$-nitroso guanidine), and NMU ( $N$-nitroso- $N$-methylurea) were purchased from Sigma. They were dissolved in DDW and supplied to sporangia of $P$. infestans to induce mutations for resistance to MFX or CAAs at periods/concentrations indicated in Table 1.

Fungicides. Formulated fungicides mandipropamid (MPD, 25\% SC), dimethomorph (DMM, 50\% WP), and mefenoxam (MFX, 48\% WP) were supplied by Syngenta Crop Protection, Stein, Switzerland. Formulated iprovalicarb (IPRO, $50 \%$ WG) was supplied by Lidor Chemicals, Ra' anana, Israel.

Sensitivity to CAAs or MFX and mating type. Sensitivity of the wild-type isolates to CAA fungicides was tested as described before (5). Briefly, sporangia $(2 \times$ $\left.10^{3} / \mathrm{ml}\right)$ were mixed $(1: 1, \mathrm{vol} / \mathrm{vol})$ with CAAs of 0.001 to $100 \mu \mathrm{g} / \mathrm{ml}$ ( $\times 10$ step dilutions) and inoculated onto the lower surface of detached leaflets of potato ('Bintje') or tomato ('Baby', or 'ZH' inbred line), $10 \times 10 \mu \mathrm{l}$ droplets/leaflet, 5 leaflets/dose. Percentage of sporulating leaflet area was recorded at 7 days postinoculation (dpi). Sensitivity to MFX was tested as described by Rubin and Cohen (23). Mating type of wild-type and mutant isolates was determined by coinoculation with reference A1 and A2 isolates as described by Rubin and Cohen (23).

Mutagenesis. Table 1 describes the experiments conducted with sporangia of $P$. infestans to induce resistance against CAA or MFX fungicides. A total of 57 experiments were performed with 10 to $50 \times 10^{6}$ sporangia/experiment with a total of 1.238 $\times 10^{9}$ sporangia. Mutations were induced by UV light (experiments 1 to 34 ) or chemical mutagenic agents (experiments 35 to 57). Intact sporangia were exposed to mutagens in the following ways: in RDA cultures (experiments 1 to $12 ; 19$ to 21 ), in situ on sporulating leaves of potato (experiments 13 to 18 ), or as detached sporangia in double-distilled water (DDW) collected from RDA cultures or from late blight-infected potato or tomato leaves (experiments 22 to 57). Three sources of UV light (one $302 \mathrm{~nm}$ and two $254 \mathrm{~nm}$ ) were used, according to availability (see below).

UV light $302 \mathrm{~nm}$. RDA cultures (11 to 14 days old, in different experiments) of isolates 96 or 28 were exposed (no lids) while on ice to UV light $(302 \mathrm{~nm}, 6 \times 25 \mathrm{~W}$ lamps, at a distance of $10 \mathrm{~cm}$ ) for $30 \mathrm{~min}$. The irradiated sporangia were collected into DDW, washed over an 8- $\mu \mathrm{m}$ Millipore membrane (Millipore Corp., Bedford, MA, USA), suspended in $8 \mathrm{ml}$ DDW (about 15 $\times 10^{6}$ sporangia), divided into $0.5-\mathrm{ml}$ aliquots, mixed (1:1 vol/vol) with $1,10,100$ $\mu \mathrm{g} / \mathrm{ml}$ of MFX, DMM, or MPD (final concentrations $0.5,5,50 \mu \mathrm{g} / \mathrm{ml}$ ) and inoculated (50 droplets, $20 \mu \mathrm{l}$ each leaflet) onto detached leaves of potato 'Bintje' in $20 \times$ $20 \times 3 \mathrm{~cm}$ Nunk dishes. Late blight lesions that developed at $7 \mathrm{dpi}$ on fungicidetreated leaves were considered to be putative resistant mutants (generation 0). Sporangia were collected with a sterile camelhair brush from each such lesion into DDW and tested for sensitivity/resistance to CAA and MFX on potato leaves as de-

Table 1. Description of the experiments conducted to induce resistance against mefenoxam (MFX) and carboxylic acid amides (CAAs) in Phytophthora infestans $^{\mathrm{a}}$

\begin{tabular}{|c|c|c|c|c|c|c|c|c|c|c|c|}
\hline \multirow[b]{2}{*}{ Experiment } & \multirow[b]{2}{*}{ Mutagen } & \multirow[b]{2}{*}{ Isolate $^{b}$} & \multirow{2}{*}{$\begin{array}{l}\text { No. of } \\
\text { exp. }\end{array}$} & \multirow{2}{*}{$\begin{array}{c}\text { Sporangia/ } \\
\text { exp. } \times 10^{6}\end{array}$} & \multirow{2}{*}{$\begin{array}{c}\text { Sporangia } \\
\text { in (on) }\end{array}$} & \multicolumn{2}{|c|}{ Exposure } & \multirow{2}{*}{$\begin{array}{c}\text { Total } \\
\text { sporangia } \\
\times 10^{6}\end{array}$} & \multicolumn{2}{|c|}{ Mutants resistant to } & \multirow{2}{*}{$\begin{array}{l}\text { Mutants } \\
\text { changed } \\
\text { A1 to A2 }\end{array}$} \\
\hline & & & & & & Min/energy & Conc. & & MFX & CAAs & \\
\hline $1-7$ & UV $302 \mathrm{~nm}$ & 96 & 7 & 15 & Culture & $3 \times 30$ & na & 105 & 2 & 0 & 2 \\
\hline $8-12$ & & 28 & 5 & 15 & Culture & $3 \times 30$ & na & 75 & 4 & 0 & \\
\hline $13-15$ & & 96 & 3 & 10 & Leaves & 30 & na & 30 & 0 & 0 & \\
\hline $16-18$ & & 28 & 3 & 10 & Leaves & 30 & na & 30 & 0 & 0 & \\
\hline $19-20$ & UV $254 \mathrm{~nm}$ & 96 & 2 & 15 & Culture & 120 & na & 30 & 0 & 0 & \\
\hline $20-21$ & & 96 & 2 & 6 & Culture & $\begin{array}{c}12.5-1,000 \\
\mathrm{~mJ} / \mathrm{cm}^{2}\end{array}$ & na & 108 & 0 & 0 & \\
\hline $22-26$ & & Mix. ${ }^{c}$ & 5 & 50 & Suspension & $3 \times 120$ & na & 250 & na & 0 & \\
\hline $27-30$ & UV254+EB & $\mathrm{AD}$ & 4 & 10 & Suspension & 120 & $0.5-5 \mu \mathrm{g} / \mathrm{ml}$ & 40 & 0 & 0 & \\
\hline $31-34$ & & $367^{d}$ & 4 & 10 & Suspension & 120 & $0.5-5 \mu \mathrm{g} / \mathrm{ml}$ & 40 & na & 0 & \\
\hline 35 & EMS & 96 & 1 & 10 & Suspension & Continuous & $0.20 \%$ & 10 & 0 & 0 & \\
\hline 36 & & 96 & 1 & 30 & Suspension & Continuous & $0.20 \%$ & 30 & 0 & 0 & \\
\hline $37-39$ & & 96 & 3 & 10 & Suspension & Continuous & $0.07-0.33 \%$ & 30 & 0 & 0 & \\
\hline 40 & & 28 & 1 & 10 & Suspension & Continuous & $0.20 \%$ & 10 & 0 & 0 & \\
\hline 41 & & 408 & 1 & 50 & Suspension & Continuous & $0.20 \%$ & 50 & 1 & 0 & \\
\hline 42 & & 409 & 1 & 50 & Suspension & Continuous & $0.20 \%$ & 50 & 8 & 0 & \\
\hline 43 & & 410 & 1 & 50 & Suspension & Continuous & $0.20 \%$ & 50 & 4 & 0 & \\
\hline 44 & & 411 & 1 & 50 & Suspension & Continuous & $0.20 \%$ & 50 & 0 & 0 & \\
\hline 45 & & 412 & 1 & 50 & Suspension & Continuous & $0.20 \%$ & 50 & 0 & 0 & 1 \\
\hline $46-47$ & & $\operatorname{Mix} .^{c}$ & 2 & 50 & Suspension & Continuous & $0.1-5 \%$ & 100 & na & 0 & \\
\hline 48 & & $\operatorname{Mix} .^{c}$ & 1 & 20 & Suspension & 120 & $0.60 \%$ & 20 & na & 0 & \\
\hline $49-50$ & EMS+UV254 & $\operatorname{Mix} .^{c}$ & 2 & 10 & Suspension & 120 & $0.50 \%$ & 20 & na & 0 & \\
\hline $51-53$ & MNNG & 96 & 3 & 10 & Suspension & Continuous & $5-500 \mu \mathrm{g} / \mathrm{ml}$ & 30 & 0 & 0 & \\
\hline \multirow[t]{2}{*}{$54-57$} & NMU & 96 & 3 & 10 & Suspension & Continuous & $5-500 \mu \mathrm{g} / \mathrm{ml}$ & 30 & 0 & 0 & \\
\hline & Total & & 57 & & & & & 1,238 & 19 & 0 & 3 \\
\hline
\end{tabular}

a EMS = ethyl methan sulfonate; EB = ethidium bromide; $\mathrm{MNNG}=$ methyl- $N$-nitroso guanidine; NMU = $N$-nitroso- $N$-methylurea; na = not applicable.

b All individual isolates were A1, except 28 and 367 , which were A2.

c Mixture of 100 field isolates and 200 hybrid isolates, many were resistant to MFX.

d A field isolate, naturally resistant to MFX. 
scribed above. Stability of resistance was tested by repeated inoculations of potato leaves with sporangia mixed with CAAs, MFX, or water and inoculated onto potato leaflets (generation 1). Putative resistant mutants propagated on untreated leaves were irradiated two more times in generations 2 and 3. The experiment was repeated seven times with isolate 96 (experiments 1 to 7 , Table 1) and five times with isolate 28 (experiments 8 to 12, Table 1). Altogether, six resistant isolates were recovered. Two mutant isolates, 96/1 and $96 / 2$, were recovered from isolate 96 , both expressing stable resistance to MFX (in vivo and in vitro) but transient resistance to CAAs. They both were converted to the A2 mating type. Four mutant isolates, $28 / 1,28 / 2,28 / 3,28 / 4$, were recovered from isolate 28 , all showing stable resistance to MFX (in vivo and in vitro) but transient resistance to CAAs (all kept their A2 mating type).

In experiments 13 to 18 , detached potato leaves in plates were drop-inoculated with isolate 96 or 28 . At $7 \mathrm{dpi}$, when profuse sporulation occurred on leaves, plates were placed on ice, lids were removed, and leaves were exposed for $30 \mathrm{~min}$ to UV light. Sporangia were thereafter collected, washed, mixed with fungicides, and inoculated onto potato leaves as described above.

UV light $254 \mathbf{~ n m}$. In experiments 20 to 21 (Table 1), RDA cultures of isolate 96 were exposed, with no lids, to UV light of $254 \mathrm{~nm}$ of various doses (5 plates/dose of 12.5 to $1,000 \mathrm{~mJ} / \mathrm{cm}^{2}$ ) using UV Stratagene 2400 chamber (Stratagene, La Jolla, CA, USA). Irradiation lasted from 3 to 270 s. Nonirradiated cultures served as controls. Two days after irradiation, sporangia were collected with a jet of sterile water, mixed with water (as control), CAAs, or MFX, and inoculated onto detached potato leaves.

Detached sporangia in experiments 22 to 26 (Table 1) were mutated as follows: $50 \mathrm{ml}$ of sporangial suspension in DDW $\left(50 \times 10^{6}\right.$ sporangia) composed of 300 isolates (100 field isolates and $200 \mathrm{~F} 1$ hybrids of field isolates $[3,14])$ in a $15-\mathrm{cm}$ petri dish were irradiated while on ice (no lid) with UV $254 \mathrm{~nm}$ for $2 \mathrm{~h}$ (BioRad, $8 \mathrm{~W}$ lamp, at a distance of $10 \mathrm{~cm}$ ). Sporangia were thereafter diluted in water and sprayinoculated onto 50 detached tomato leaves (in moistened $20 \times 20 \times 3 \mathrm{~cm}$ Nunk dishes) previously treated with $50 \mu \mathrm{g} / \mathrm{ml}$ of MPD, DMM, or IPRO (MFX was excluded because many isolates in the mixture were resistant to MFX). Untreated leaves served as control. At $7 \mathrm{dpi}$, sporangia (generation 0) were collected from lesions produced on the CAA-treated leaves, centrifuged $3,000 \times g$ for $10 \mathrm{~min}$, resuspended in DDW, and drop-inoculated onto 10 untreated leaves and 10 leaves sprayed with $5 \mu \mathrm{g} / \mathrm{ml}$ of that CAA on which they were developed (generation 1). To see if the transient resistance is maintained longer in the absence of a challenge with CAA, sporangia were collected at 7 dpi from control-treated leaves or CAAtreated leaves and inoculated onto new untreated leaves or leaves spray-treated with $5 \mu \mathrm{g} / \mathrm{ml}$ of the respective CAA (generation 2). The same procedure was followed during eight generations. To increase the probability that mutants with transient resistance will acquire stable CAA resistance, sporangia were collected from treated leaves at generation 0 , propagated on untreated tomato leaves, and $10 \times$ $10^{6}$ sporangia were irradiated again (second irradiation). The same procedure was repeated once again (third irradiation). Resistance was examined during eight asexual generations by inoculating the sporangia produced after each irradiation regime onto CAA-treated $(5 \mu \mathrm{g} / \mathrm{ml})$ or untreated leaves (10 leaves/treatment). In all experiments, sporangia were washed by centrifugation $\left(3000 \times g, 10 \mathrm{~min}, 4^{\circ} \mathrm{C}\right)$ before being used for subsequent inoculations.

Ethidium bromide+UV light $254 \mathbf{~ n m}$. Isolates AD and 367 were each mutated as follows (experiments 27 to 34, Table 1): sporangia $\left(10 \times 10^{6}\right)$ were collected from infected tomato leaves into DDW, washed over an 8- $\mu \mathrm{m}$ Millipore membrane, resuspended in $10 \mathrm{ml}$ of $0.5,1,2$, or $5 \mu \mathrm{g} / \mathrm{ml}$ ethidium bromide in a petri dish, and exposed (no lid) while on ice to UV light (254 nm, 8W lamp) at a distance of $10 \mathrm{~cm}$ for $2 \mathrm{~h}$. Sporangia were thereafter mixed with fungicides and inoculated onto tomato leaves. Four such experiments were done with each isolate (Table 1), from which two putative mutants $\mathrm{ADm}$ and $367 \mathrm{~m}$ were recovered. ADm maintained its A1 mating type and sensitivity to MFX, and $367 \mathrm{~m}$ maintained its A2 mating type and resistance to MFX. Both showed transient resistance to CAAs when inoculated onto CAA-treated tomato leaves.

EMS and EMS+UV light 254. Sixteen experiments were conducted with EMS, 11 with individual isolates (experiments 35 to 45, Table 1) and 5 with mixed isolates (experiments 46 to 50, Table 1). Sporangia were mixed with EMS (of various concentrations, depending on the experiment, Table 1), or with EMS and CAAs, and inoculated onto detached tomato leaves previously treated with MFX of various concentrations. In experiment 48, sporangia were incubated in EMS for $2 \mathrm{~h}$, washed, mixed with water or CAAs, and inoculated onto tomato leaves. In experiments 49 and 50, sporangia were mixed with EMS and exposed to UV 254 for $2 \mathrm{~h}$. At 7 to $10 \mathrm{dpi}$, sporangia were collected from single-lesion mutants of late blight developed on control or CAA-treated leaves, washed, mixed with water or CAAs, and inoculated onto new detached leaves. Transfer inoculations onto controluntreated leaves, CAA-treated leaves, or leaves treated with MFX were repeated for 4 to 8 asexual generations, depending on the experiment. Isolates with transient resistance to CAAs and stable resistance to MFX were obtained in these experiments.

MNNG or NMU. Sporangia of isolate 96 were removed from RDA cultures, mixed with MNNG or NMU (of various
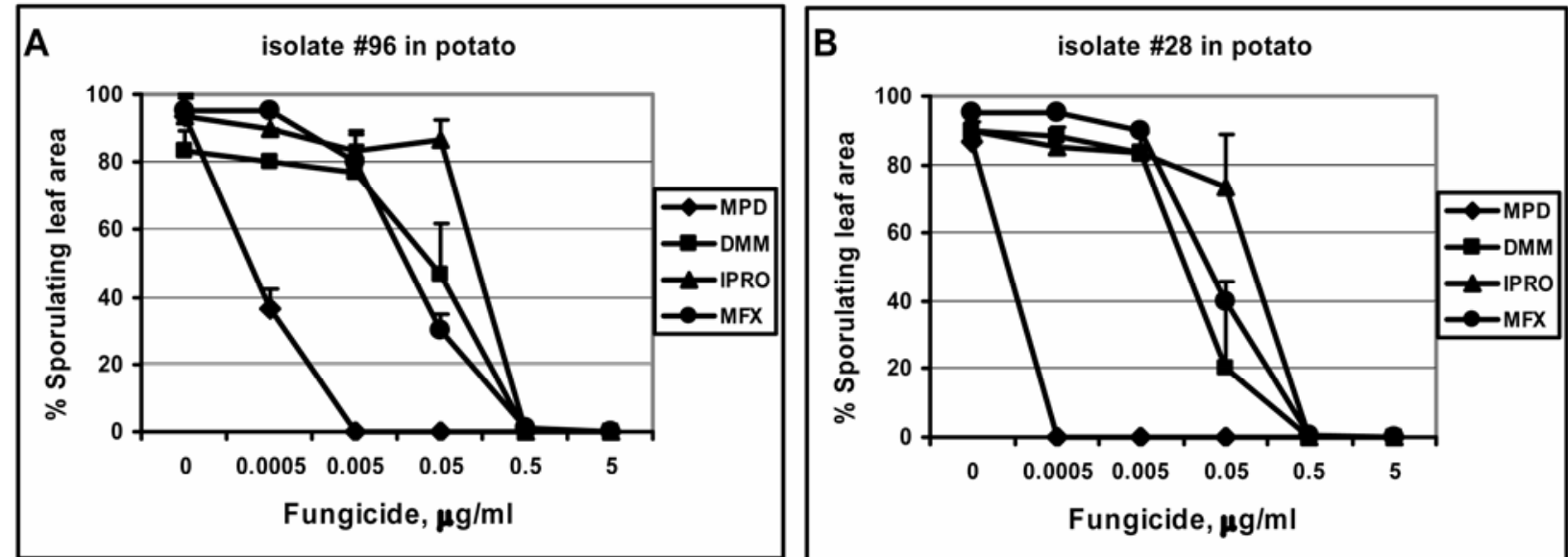

Fig. 1. Sensitivity of the wild-type isolates 96 and 28 of Phytophthora infestans to carboxylic acid amide fungicides or mefenoxam (MFX). MPD = mandipropamid, DMM = dimethomorph, IPRO = iprovalicarb. 
concentrations, experiments 51 to 57 , Table 1), mixed with water or CAAs, and inoculated onto tomato leaves. No resistant isolates were obtained (Table 1).

Crosses. Crosses were made according to the method described by Rubin and Cohen (23). A1 and A2 mutants expressing transient resistance to CAAs were crossed to possibly obtain homozygous-resistant isolates. The $\mathrm{A} 1, \mathrm{MFX}$-sensitive isolate ADm showing transient resistance to CAAs was crossed with six A2 mutant isolates showing stable resistance to MFX and transient resistance to CAAs: 28/1, 28/2, 28/3, 28/4, 96/2, and 367m. Crosses were done by co-inoculation of detached tomato leaves (20 leaflets/cross) with sporangial mixture $(1: 1)$ of the parental isolates. At $10 \mathrm{dpi}$, when abundant oospores were formed in the leaves, leaves were homogenized in $50 \mathrm{ml}$ of DDW, 5-ml aliquots were placed in each of ten $10-\mathrm{cm}$ petri dishes, dried-wetted twice to eliminate vegetative structures of $P$. infestans, and mixed with $20 \mathrm{ml}$ of DDW and $2 \mathrm{~g}$ of perlite. Tomato leaves (2/petri dish) were placed on top of the perlite-oospores mixture as baits for oospore infection. F1 progeny isolates having opposite mating types were similarly crossed to obtain F2 progeny. Single sporangium isolates (SSI) were produced from the F2 progeny isolates using the method described before (23). Resistance of F1 progeny and F2 SSIs to CAAs or MFX was determined in tomato leaves as described above.
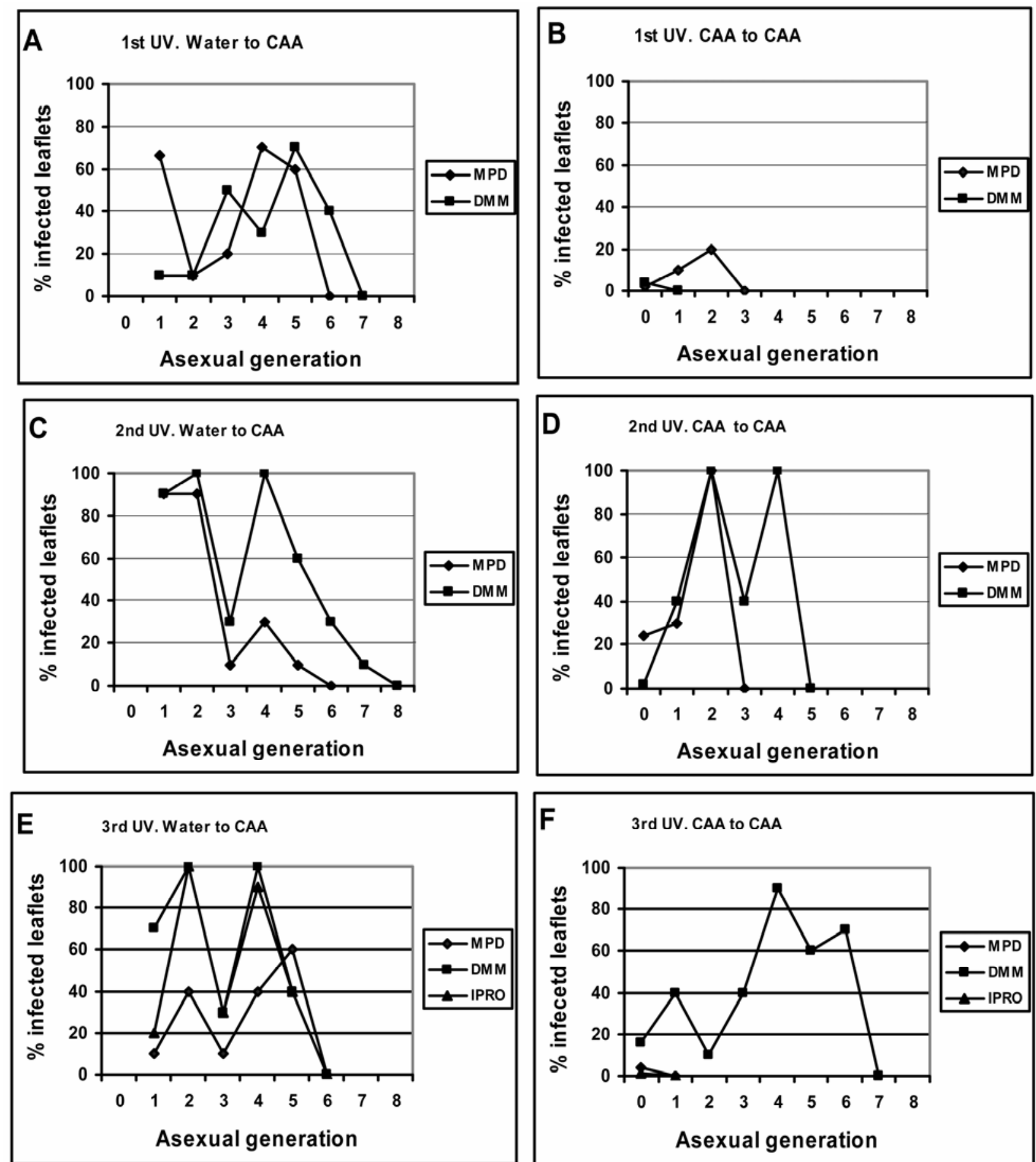

Fig. 2. Resistance of Phytophthora infestans to carboxylic acid amides (CAAs) after three consecutive UV light irradiations applied to sporangia at three consecutive asexual generations. Resistance to $5 \mu \mathrm{g} / \mathrm{ml}$ CAA was evaluated in sporangia harvested from CAA-free (controls) leaves or CAA-treated leaves at each asexual generation. Figures represent percent infected leaflets out of 20 leaflets inoculated at each generation. $\mathrm{MPD}=$ mandipropamid, DMM $=$ dimethomorph, IPRO = iprovalicarb. 


\section{RESULTS}

Sensitivity of wild-type isolates to CAAs and MFX. The wild-type isolates 96 and 28 were highly sensitive to CAAs and MFX: when inoculated onto fungicide-treated potato leaves, total inhibition of infection occurred in the presence of 0.0005 to $0.005 \mu \mathrm{g} / \mathrm{ml} \mathrm{MPD,} \mathrm{or} 0.5 \mu \mathrm{g} / \mathrm{ml}$ DMM, IPRO, or MFX (Fig. 1A and B). Similar sensitivities to CAAs and MFX were measured in isolates $408,409,410$, 411 , and 412.

The effect of UV light and/or chemical mutagens on development of resistance. Table 1 summarizes the results from 57 mutagenesis experiments conducted to induce mutations in $P$. infestans against CAAs or MFX. The results indicate that mutagenesis may induce stable resistance against MFX (see below), but not against CAAs. In repeated experiments, UV light and/or chemical mutagenic agents were applied to attached or detached sporangia (total of $1.238 \times 10^{9}$ ), in culture or on leaves, but no stable resistance to CAAs was recovered. This was true for 9 individual isolates as well as for isolate mixtures. Nonetheless, in many experiments, CAAresistant mutants (infecting leaves treated with up to $50 \mu \mathrm{g} / \mathrm{ml} \mathrm{CAA}$, in different experiments) did show up at generation 0 (using sporangia immediately after mutagenesis treatment). However, representative data (Fig. 2A to F) from experiments 22 to 26 (Table 1) show the nature of resistance to CAAs: resistance was erratic and diminished in the next inoculation transfer (Fig. 2B and F) or after a few inoculation transfers (up to 8 transfers, Fig. 2C) on tomato leaves.

In experiments 1 to 7 with isolate 96 , a shift from A1 to A2 mating type was detected in two mutants (Table 1), indicating that UV light may induce mutation in $P$. infestans. A similar shift occurred also with isolate 412 (experiment 45, Table 1).

UV light or EMS applied to sporangia of isolates 96 and 28 induced stable resistance to MFX but transient resistance against CAAs. Sporangia were collected from UV-irradiated RDA cultures $(302 \mathrm{~nm}$, $6 \times 25$ W lamps, $30 \mathrm{~min}$ ), mixed with fungicides, and inoculated onto potato leaves. Forty lesions were produced with $50 \mu \mathrm{g} / \mathrm{ml}$ MFX in isolate 96, 12 lesions with $5 \mu \mathrm{g} / \mathrm{ml}$ MFX in isolate 28, and a total of 17 lesions with 0.5 to $50 \mu \mathrm{g} / \mathrm{ml}$ CAAs (Fig. 3A and $\mathrm{B}$, Table 1 , experiments 1 to 12 ), suggesting the evolution of fungicide-resistant mutants. However, all 17 mutants developed on CAA-treated leaves at generation 0 failed to infect CAA-treated leaves in the subsequent transfer inoculation (generation 1). In contrast, those that developed on leaves treated with 5 or $50 \mu \mathrm{g} / \mathrm{ml}$ MFX were all infective to leaves treated with 50 $\mu \mathrm{g} / \mathrm{ml}$ MFX. The sporangia produced on MFX-treated leaves were collected and inoculated (generation 2) onto untreated control, DMM, MPD, or MFX-treated leaves. Sporulating lesions developed in control leaves and leaves treated with MFX (up to $50 \mu \mathrm{g} / \mathrm{ml}$ ), but not in leaves treated with DMM or MPD of $0.5,5$, or 50 $\mu \mathrm{g} / \mathrm{ml}$ (Fig. 4), suggesting that UV light of $302 \mathrm{~nm}$ induced stable resistance against MFX (resistance factor $>1,000$ ) but not against CAAs.

Sporangia of the mutant isolates 96 and 28 were collected from MFX-treated leaves (about $10 \times 10^{6}$ sporangia each, in $10 \mathrm{ml}$ water), irradiated again with UV 302 $\mathrm{nm}$ for $30 \mathrm{~min}$ (second irradiation), and inoculated onto MFX- or CAA-treated leaves. All control leaves and leaves treated with $\operatorname{MFX}(0.5,5,50 \mu \mathrm{g} / \mathrm{ml})$ showed massive sporulation of $P$. infestans, whereas leaflets treated with CAAs of 0.5 , 5 , or $50 \mu \mathrm{g} / \mathrm{ml}$ were not infected. Resistance to 5 to $50 \mu \mathrm{g} / \mathrm{ml} \mathrm{CAAs} \mathrm{reappeared} \mathrm{in}$ leaves inoculated with sporangia that were collected from MFX-treated leaves and irradiated once again (third irradiation, Fig. 3C and D). However, whereas resistance to MFX was stable, consistently expressed upon transfer inoculations, resis-
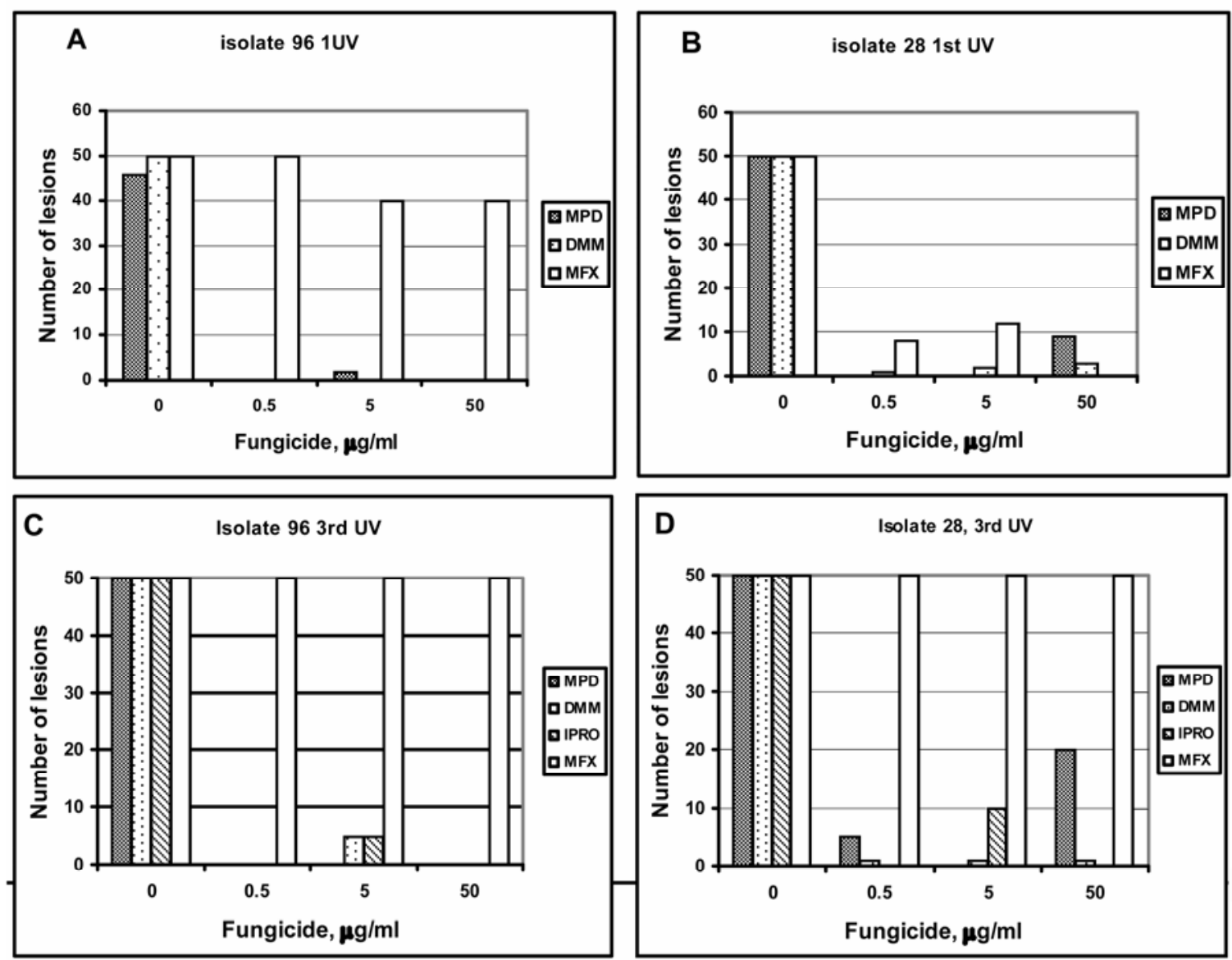

Fig. 3. Mutagenesis of two wild-type isolates of Phytophthora infestans for resistance to carboxylic acid amides (CAAs) and mefenoxam (MFX). Intact sporangia in RDA cultures were irradiated with UV $302 \mathrm{~nm}$ for $30 \mathrm{~min}$, mixed with a fungicide and inoculated onto potato leaflets. MPD = mandipropamid, $\mathrm{DMM}=$ dimethomorph, $\mathrm{IPRO}=$ iprovalicarb. 
tance to CAAs diminished after one or two inoculation transfers (data not shown). Four mutants of isolate 28 and two mutants of isolate 96 , all resistant to MFX (28/1, 28/2, 28/3, 28/4, 96/1, and 96/2, all A2) were resistant in MFX-amended RDA cultures $(100 \mu \mathrm{g} / \mathrm{ml})$, but not in CAAamended $(1.5 \mu \mathrm{g} / \mathrm{ml})$ RDA cultures. They were maintained for further genetic studies (see Crosses below).

In experiments 20 to 21 (Table 1), no late blight lesions developed when sporangia were mixed with CAAs or MFX. The cultures were allowed to regrow for an additional 2 days (total of 4 days after irradiation), sporangia were collected into DDW from all plates, mixed, washed over
8- $\mu \mathrm{m}$ Millipore membrane, resuspended in DDW, and drop-inoculated onto detached potato leaves. These sporangia were highly infective to untreated potato leaves and produced 8 and 1 lesions in leaves treated with 5 or $50 \mu \mathrm{g} / \mathrm{ml}$ DMM, respectively, and 1 and 0 lesions in leaves treated with 5 or $50 \mu \mathrm{g} / \mathrm{ml}$ MPD, respectively. They produced HR lesions in the presence of MFX. No late blight lesions developed in CAAor MFX-treated leaves upon further transfer inoculations, suggesting that no resistant mutants have evolved. Cultures irradiated with UV of 12.5 to $1,000 \mathrm{~mJ} / \mathrm{cm}^{2}$ and soon subcultured on CAA-amended RDA showed no growth on 2.5 or $5 \mu \mathrm{g} / \mathrm{ml}$ MPD (DMM and MFX were not tested).
EMS applied to detached sporangia of isolates 408 to 412 (experiments 41 to 45 , Table 1) induced stable resistance to MFX but transient resistance against CAAs. EMS-treated sporangia of isolates 408, $409,410,411$, and 412 induced the formation of $5,6,1,2$, and 4 sporulating lesions in 40 leaves treated with $5 \mu \mathrm{g} / \mathrm{ml}$ DMM, and $3,7,3,2$, and 2 sporulating lesions in 40 leaves treated with $5 \mu \mathrm{g} / \mathrm{ml}$ MPD. The 18 resistant isolates recovered from DMM and the 17 resistant isolates recovered from MPD were tested for mating type, and each was transfer-inoculated onto leaves treated with $5 \mu \mathrm{g} / \mathrm{ml}$ of the respective CAA they were derived from. The ability of isolates $408,409,410,411$, and

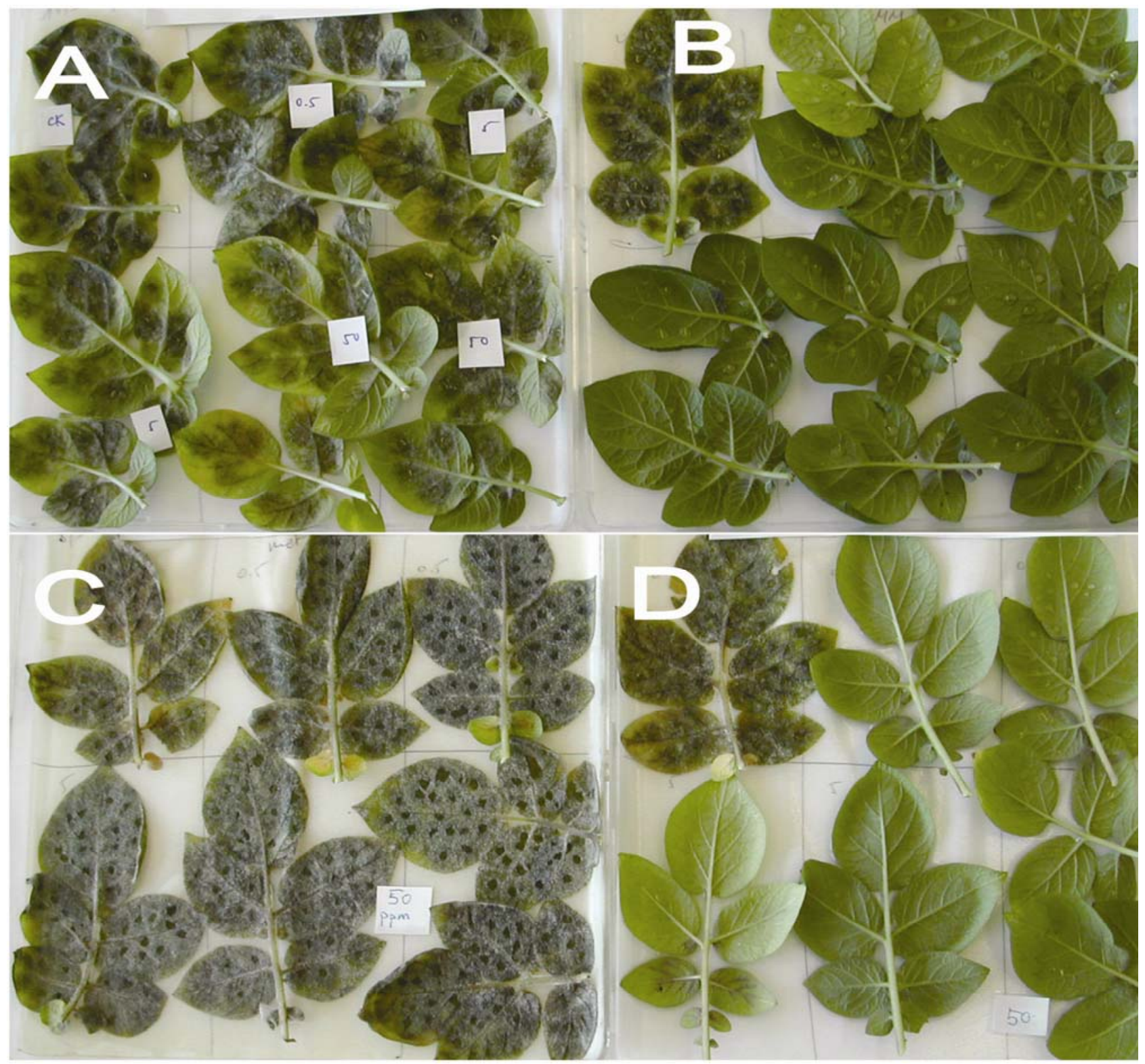

Fig. 4. Mutagenesis of two wild-type isolates of Phytophthora infestans for resistance to mefenoxam (MFX) and carboxylic acid amides (CAAs). Intact sporangia in RDA cultures were irradiated with UV $302 \mathrm{~nm}$ for $30 \mathrm{~min}$, mixed with a fungicide, and inoculated onto potato leaflets. A, Isolate 96 , response to MFX: from top left in clockwise direction, leaves were treated with MFX of 0, 0.5, 5, 5, 50, and $50 \mu \mathrm{g} / \mathrm{ml}$ before inoculation. B, Isolate 96 , response to dimethomorph (DMM): from top left in clockwise direction, leaves were treated with DMM of $0,0.5,0.5,5,5$, and $50 \mu \mathrm{g} / \mathrm{ml}$ before inoculation. C, Isolate 28, response to MFX: from top left in clockwise direction, leaves were treated with MFX of $0,0.5,5,5,50$, and $50 \mu \mathrm{g} / \mathrm{ml}$ before inoculation. D, Isolate 28 , response to mandipropamid (MPD): from top left in clockwise direction, leaves were treated with MPD of 0, 0.5, 0.5, 5, 5, and 50 $\mu \mathrm{g} / \mathrm{ml}$ before inoculation. Photos A and B were taken at $7 \mathrm{dpi}, \mathrm{C}$ and $\mathrm{D}$ at $8 \mathrm{dpi}$. 
412 to infect DMM-treated leaves was abolished after 1, 4, 1, 2, 0 transfer inoculations, and the ability to infect MPDtreated leaves after 1, 2, 4, 4, 0 transfer inoculations, respectively.

In these experiments, EMS applied to sporangia of isolates 408, 409, 410, 411, and 412 induced the formation of $1,8,4$, 0 , and 0 late blight lesions (isolates) in inoculated leaves (20 leaves/isolate) floating on $5 \mu \mathrm{g} / \mathrm{ml}$ MFX, respectively. After six transfer inoculations on MFX-treated leaves, 1, 3, and 2 mutant isolates of 408 , 409,410 were intermediately resistant, and 0,5 , and 2 isolates were resistant to MFX, respectively (Table 1). All 13 isolates belonged to the A1 mating type, as did their wild-type parents. One mutant derived from isolate 412 changed from A1 to A2 (experiment 45, Table 1).

Crosses between mutants showing transient resistance to CAAs and stable resistance to MFX. The following six A2 mutant isolates showed stable resistance to MFX and transient resistance to CAAs: 28/1, 28/2, 28/3, 28/4, 96/2, and 367m. They were each crossed with the A1, MFX-sensitive isolate ADm that showed transient resistance to CAAs. Fifty-nine F1 progeny isolates (late blight lesions) were obtained from six crosses, 4 to 13 isolates/cross. They belonged to A1, A2, or to the unusual A1A2 mating type (Fig. 5). A1A2 isolates occurred in previous crosses of $\mathrm{A} 1$ and $\mathrm{A} 2$ (23). At generation 0, none of these 59 isolates was resistant to $\geq 1$ $\mu \mathrm{g} / \mathrm{ml}$ MPD (Fig. 6A), whereas 48, 11, and 0 were resistant to DMM at 1,5 , and 10 $\mu \mathrm{g} / \mathrm{ml}$, respectively (Fig. 6B). Forty-nine isolates were resistant $(\mathrm{R})(>10 \mu \mathrm{g} / \mathrm{ml})$ to MFX, 10 were intermediately resistant (I) ( 1 to $10 \mu \mathrm{g} / \mathrm{ml}$ ), and none was sensitive (S) $(<1 \mu \mathrm{g} / \mathrm{ml}$ ) (Fig. 6C). All F1 isolates lost their resistance to DMM at $\geq 1 \mu \mathrm{g} / \mathrm{ml}$ after 1 to 3 inoculation transfers. Thirteen sib crosses were made between F1 isolates of opposite mating types (two or three per cross). Four sib F2 progeny lesions (isolates) were obtained on tomato leaves in perlite bait cultures, two from $28 / 1 \times \mathrm{ADm}$ ( $\mathrm{F} 1$ isolates $12 \times 13=\mathrm{A} 2 \mathrm{R} \times \mathrm{A} 1 \mathrm{R}$; and $\mathrm{F} 1$ isolates $18 \times 20=\mathrm{A} 2 \mathrm{I} \times \mathrm{A} 1 \mathrm{R})$, one from 96/2×ADm (F1 isolates $43 \times 46=\mathrm{A} 1 \mathrm{R} \times \mathrm{A} 2 \mathrm{R}$ ), and one from $367 \mathrm{~m} \times \mathrm{ADm}$ ( $\mathrm{F} 1$ isolates $54 \times 57=\mathrm{A} 1 \mathrm{R} \times \mathrm{A} 2 \mathrm{R})$. Sporangia from $\mathrm{F} 2$ lesions were used to produce 25 to 40 single sporangium isolates (SSI) from each F2 progeny lesion. The sensitivities (generation 0) of 108 to $130 \mathrm{~F} 2$ SSIs to MPD, DMM, and MFX are presented in Fig. 6D, $\mathrm{E}$, and F, respectively. Resistance to DMM was more frequent than resistance to MPD. In total, 97,16 , and $6 \%$ of the F2 SSI isolates were resistant to DMM at 1, 5, and 10 $\mu \mathrm{g} / \mathrm{ml}$, respectively (Fig. $6 \mathrm{E}$ ), as against $24,1.5$, and $0 \%$, which were resistant to MPD at the corresponding concentrations (Fig. 6D).

These F2 progeny isolates were kept in growth chambers at $12^{\circ} \mathrm{C}$ by weekly trans- fer inoculations of untreated tomato leaves to examine whether or not they hold their resistance in the absence of challenge with DMM or MPD. After 12 such transfer inoculations, sporangia of all SSI isolates were bulked together, mixed with each CAA, inoculated onto detached tomato leaves, 20 leaflets/fungicide/dose treatment, and the proportion of infected leaves showing sporulation was counted after 7 days. The pathogen maintained resistance to $1 \mu \mathrm{g} / \mathrm{ml}$ ( $40 \%$ of the leaflets infected) and $10 \mu \mathrm{g} / \mathrm{ml}(10 \%)$ DMM and to $1 \mu \mathrm{g} / \mathrm{ml}$ (15\%) MPD. No infection occurred with $10 \mu \mathrm{g} / \mathrm{ml}$ MPD. Sporangia collected from such CAA-treated leaves failed to infect new leaves treated with $1 \mu \mathrm{g} / \mathrm{ml}$ of either CAA)

Resistance to MFX is shown in Figure $6 \mathrm{~F}$. About 40, 57, and 3\% of the isolates were resistant, intermediately resistant, and sensitive to MFX, respectively. Crosses $12 \times 13$ and $43 \times 46$, in which both parents were $\mathrm{R}$, produced $\mathrm{R}$ and I offspring isolates, whereas cross $18 \times 20$, in which one parent was I and the other R, produced S, I, and R offspring isolates (Fig. 6F).

\section{DISCUSSION}

Isolates of $P$. infestans resistant to metalaxyl evolved in nature soon after the introduction of metalaxyl to the market $(3,8,17)$. This rapid buildup of resistance resulted from selection of naturally occurring resistant subpopulations imposed by metalaxyl on the pathogen (17). On the other hand, in spite of the long use of the CAA fungicide dimethomorph (DMM) in nature, and the frequent applications (5 years) of the newly developed CAA mandipropamid in trial sites in Europe and Israel (6), no mutants of $P$. infestans resistant to CAA fungicides have been detected. In contrast to the situation with $P$. infestans, resistance in $P$. viticola to all CAAs has been reported in European vineyards in recent years (9). It was assumed that resistance in $P$. viticola, known to be controlled by two recessive nuclear genes, occurred in nature and, due to the annual sexual reproduction typical to this pathogen, resistance was fixed in the populations (9).

The reasons for no occurrence of CAA resistance in $P$. infestans in nature are not clear. They may be attributed to the failure of mutagenic agents (UV light, chemicals) to induce mutations in the pathogen, the need for multiple-loci mutations, rare occurrence of oospores in nature, the poor fitness of resistant mutants, or all reasons combined.

The purpose of this study was to explore whether or not resistance to CAAs and MFX in $P$. infestans may evolve by artificial mutagenesis with UV light or chemicals. We used sporangia in our mutagenesis studies because they are the principle vehicle driving epidemics of late blight in nature and as such are likely to be exposed to direct UV irradiation and/or chemicals. Mutants were selected by inoculating mixtures of the mutated sporangia with CAA or MFX onto potato (or tomato) leaves in order to detect mutants whose pathogenicity was not impaired.

The data show that mutagenic agents applied to sporangia $P$. infestans often induced the formation of late blight lesions in the presence of CAA or MFX. However,

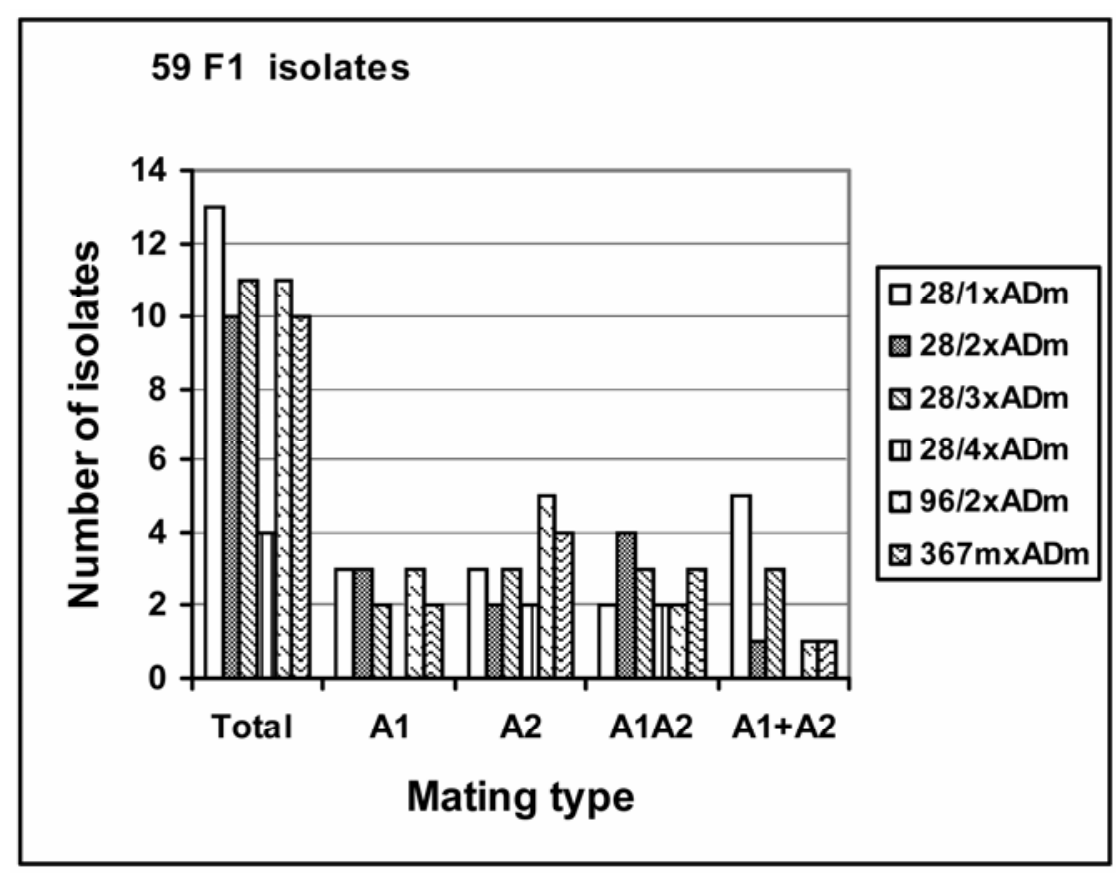

Fig. 5. Mating type of $59 \mathrm{~F} 1$ isolates obtained from six crosses between mefenoxam (MFX)-resistant parental isolates and one MFX-sensitive parental isolate. All parents exhibited transient resistance to carboxylic acid amides (CAAs). 
resistance induced against MFX by either UV $302 \mathrm{~nm}$ or EMS was stable and inherited, whereas the resistance induced against CAAs was erratic and transient. In 12 experiments, in which a total of $1.8 \times$ $10^{8}$ sporangia were treated with UV 302 $\mathrm{nm}$, six mutants highly resistant to MFX were obtained (frequency of $3.3 \times 10^{-8}$ ). This resistance was stable in vitro and in planta in the presence or absence of MFX. In another 14 experiments, in which a total of $3.3 \times 10^{8}$ sporangia were treated with EMS, 13 mutants, resistant or intermediately resistant to MFX, were obtained (frequency of $3.9 \times 10^{-8}$ ). On the other hand, mutants (produced by treating sporangia with UV light and/or chemical agents) resistant to CAAs in planta at generation 0 lost infectivity within one or a few transfer inoculations onto new CAAtreated leaves. Similar results were reported for mutants resistant to DMM $(2,7,24)$ and flumorph (27).

Based on the data published for $P$. viticola (9), we assumed that resistance to CAAs in $P$. infestans might also be controlled by two or more recessive genes. This assumption corroborates with the proposal raised by Bagirova et al. (2) that polygenic (additive) recessive genes con- trol resistance against DMM in $P$. infestans. If true, partial resistance is likely to appear after several steps of mutagenesis, and stable resistance only after sexual recombination of heterozygous, sensitive mutants.

In the present study, progeny isolates obtained from crosses made between mutant isolates with transient resistance to CAAs did not differ from their parents in response to CAAs. They too showed transient expression of resistance to CAAs in planta and failed to grow in CAA-amended RDA cultures. These crosses, made between MFX-sensitive and MFX-resistant
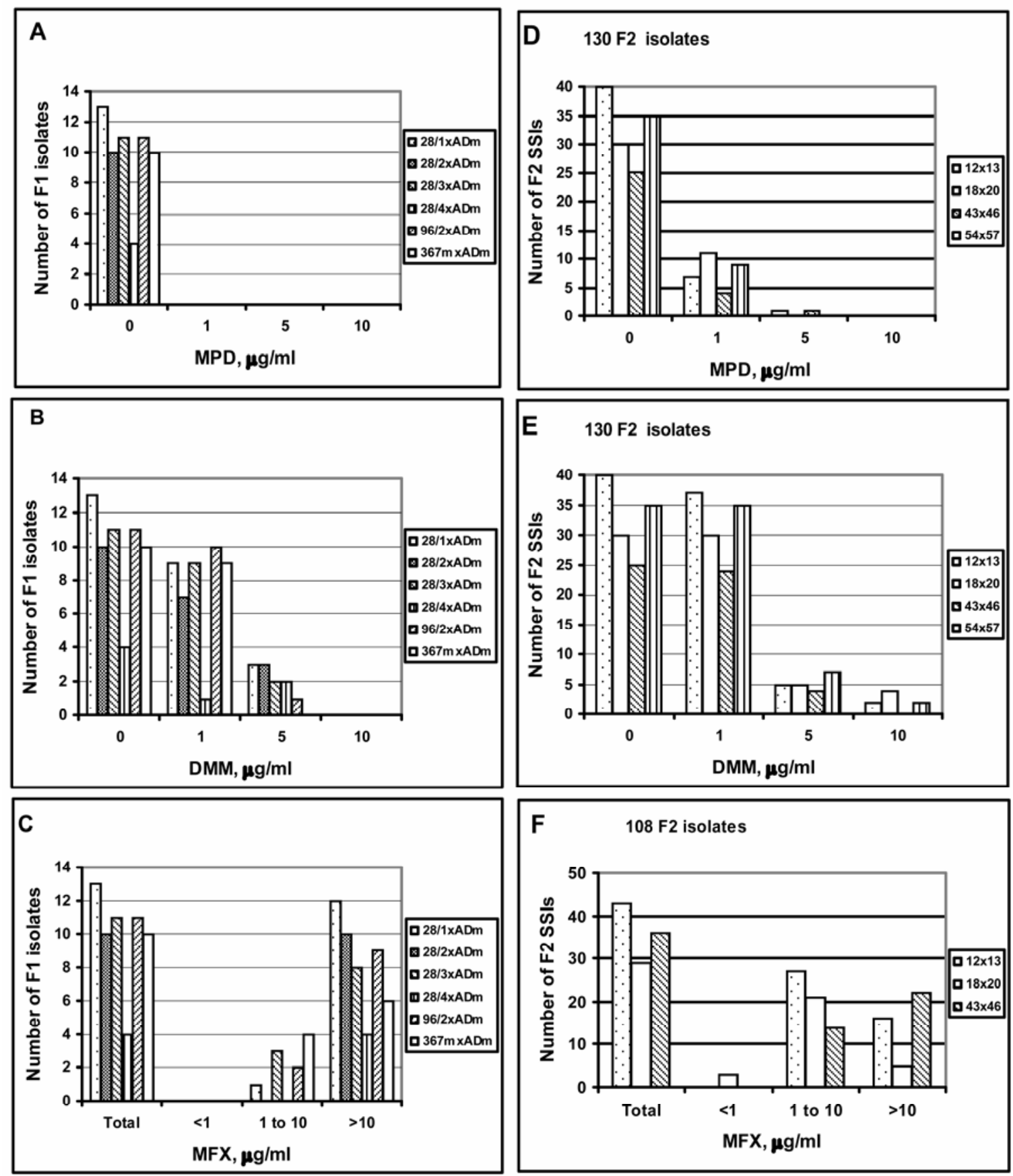

Fig. 6. Resistance of F1 and F2 progeny isolates of Phytophthora infestans derived from crosses between mutant parents expressing stable resistance to mefenoxam (MFX) and transient resistance to carboxylic acid amides (CAAs). MPD = mandipropamid, DMM = dimethomorph. SSIs $=$ single sporangium isolates. 
mutants (both expressing transient resistant to CAAs), segregated in response to MFX, producing mainly resistant and intermediately resistant isolates, indicating that the parental mutant isolates carried a dominant gene(s) for resistance to MFX. This indication is supported by the fact that parental mutants were already resistant to MFX at generation 0 after mutagenesis of sporangia. Similar results were obtained by Bagirova et al. (2) with metalaxyl. They showed that crossing of two DMMresistant reverent mutants, one metalaxylresistant and the other metalaxyl-sensitive, produced 22 resistant and 3 intermediately resistant F1 offspring isolates. They suggested that a dominant gene controls resistance against metalaxyl in their mutants. In nature, MFX resistance is usually controlled by a single, partially dominant gene and several minor modifier genes (8). One study reported on a dominant gene for resistance to metalaxyl (20). It might be that artificially produced MFX-resistant mutants carry a gene(s) for resistance different from that in the naturally occurring MFX-resistant isolates. If true, crosses between artificial resistant mutants and natural resistant isolates should yield sensitive progeny isolates. This hypothesis is currently being tested.

The data provided in this study stand in accordance with earlier studies reporting on the failure to mutate $P$. infestans for resistance to DMM or flumorph $(2,24,27)$. They also explain our failure to detect resistant isolates among many isolates collected in nature in Israel or Europe (6). The data, however, disagree with the recent findings of Ziogas et al. (29), who reported on multidrug resistance in UVproduced mutants of $P$. infestans. Their mutants were resistant not only to CAAs but also to chemically unrelated fungicides, including metalaxyl, cymoxanil, cyazofamid, and even chlorothalonil.

Our failure to produce stable CAAresistant mutants in the laboratory, together with the failure to enforce the selection of resistant isolates by heavy and continuous sprays of CAAs in the field $(6,24,25)$, suggest that CAA-resistant mutants of $P$. infestans are unlikely to appear soon in nature. There might be several hypothetical reasons for this no-show of stable CAA resistance in $P$. infestans, including the following: (i) Multiple targets or multiple loci are associated with the anti-oomycete activity of CAAs. Additive mutations in one or several genes are involved in resistance, and many selection cycles are required for achieving stable resistance. (ii) Inheritance of all or some of the resistant loci is recessive as in Plasmopara viticola (7), thus requiring repeated sexual recom- bination cycles, not very common in $P$. infestans in nature, to be expressed phenotypically. (iii) Resistance in P. infestans, unlike in Plasmopara viticola, might be lethal or associated with severe penalties such as reduced growth and fitness, which compared with wild-type populations. (iv) $P$. infestans, unlike Plasmopara viticola, is equipped with an efficient mechanism capable of deleting resistant loci from the genome, or is lacking a mechanism to keep such loci intact in the genome.

\section{LITERATURE CITED}

1. Albert, G., Thomas, A., and Guehne, M. 1991. Fungicidal activity of dimethomorph on different stages in the life cycle of Phytophthora infestans and Plasmopara viticola. ANPP - Int. Conf. Plant Dis., 3rd, Bordeaux.

2. Bagirova, S. F., Li, A. Z., Dolgova, A. V., Elansky, S. N., Shaw, D. S., and Dyakov, Y. T. 2001. Mutants of Phytophthora infestans resistant to dimethomorph fungicide. J. Russian Phytopathol. 2:19-24.

3. Cohen, Y. 2002. Populations of Phytophthora infestans in Israel underwent three major genetic changes during 1983 to 2000. Phytopathology 92:300-307.

4. Cohen, Y., Baider, A., and Cohen, B.-H. 1995. Dimethomorph activity against oomycete fungal plant pathogens. Phytopathology 85:15001506.

5. Cohen, Y., and Gisi, U. 2007. Differential activity of carboxylic acid amides fungicides against various developmental stages of Phytophthora infestans. Phytopathology 97:12741283.

6. Cohen, Y., Rubin, E., Hadad, T., Gotlieb, D., Sierotzki, H., and Gisi, U. 2007. Sensitivity of Phytophthora infestans to mandipropamid and the effect of enforced selection pressure in the field. Plant Pathology 56: 836-842.

7. Dereviagina, M. K., Elanskij, S. N., and Diakov, Y. T. 1999. Resistance of Phytophthora infestans to the dimethomorph fungicide. Mikologiya Fitopatol. 33:208-213.

8. Gisi, U., and Cohen, Y. 1996. Resistance to phenylamide fungicides: A case study with Phytophthora infestans involving mating type and race structure. Annu. Rev. Phytopathol. 34:549-572.

9. Gisi, U., Waldner, M., Kraus, N., Dubuis, P. H., and Sierotzki, H. 2006. Inheritance of resistance to carboxylic acid amide (CAA) fungicides in Plasmopara viticola. Plant Pathol. 56:199-208.

10. Griffiths, R. G., Dancer, J., O'Neill, E., and Harwood, J. L. 2003. A mandelamide pesticide alters lipid metabolism in Phytophthora infestans. New Phytol. 158:345-353.

11. Harp, T., Cloud, G., Minton, B., and Cochran, A. 2006. Mandipropamid: A new fungicide for control of late blight and downy mildews. (Abstr.) Phytopathology 96:S185.

12. Hermann, D., Bartlett, D. W., Fischer, W., and Kempf, H. J. 2005. The behaviour of mandipropamid on and in plants. Proc. BCPC Int. Congr. Crop Sci. Technol., Glasgow, UK. 1:87-92.

13. Huggenberger, F., and Kuhn, P. J. 2006. Biological and physico-chemical properties of mandipropamid, a new fungicide for the control of oomycetes pathogens. (Abstr.) Phytopathology 96:S51.

14. Irzhansky, I., and Cohen, Y. 2006. Inheritance of resistance against Phytophthora infestans in
Lycopersicon pimpenellifolium L3707. Euphytica 149:309-316.

15. Jende, G., Steiner, U., and Dehne, H.-W. 1999. Effects of iprovalicarb (SZX 0722) on the development of Phytophthora infestans in tomato plants. Pflanzenchutz-Nachr. Bayer 52:49-60.

16. Jende, G., Steiner, U., and Dehne, H.-W. 2001 Microscopical characterization of fungicidal effects on infection structures and cell wall formation of Phytophthora infestans. Pages 83-90 in: Modern Fungicides and Antifungal Compounds. AgroConcept and Verlag, Bonn, Germany

17. Kadish, D., and Cohen, Y. 1989. Population dynamics of metalaxyl-sensitive and metalaxyl-resistant isolates of Phytophthora infestans in untreated crops of potato. Plant Pathol. 38:271-276.

18. Knauf-Beiter, G., and Hermann, D. 2005. Site of action of mandipropamid in the infection cycle of target fungi. Proc. BCPC Int. Congr. Crop Sci. Technol., Glasgow, UK. 1:99-104.

19. Lamberth, C., Cederbaum, F., Jeanguenat, A. Kempf, H. J., Zeller, M., and Zeun, R. 2006. Synthesis and fungicidal activity of N-2-(3methoxy-4-propagyloxy) phenylethyl amides. Part II: Anti-oomycetic mandelamides. Pest Manag. Sci. 62:446-451.

20. Lee, T. Y., Mizubuti, E., and Fry, W. E. 1999. Genetics of metalaxyl resistance in Phytophthora infestans. Fungal Genet. Biol. 26:118-130.

21. Matheron, M. E., and Porchas, M. 2000. Impact of azoxystrobin, dimethomorph, fluazinam, fosetyl-Al, and metalaxyl on growth, sporulation, and zoospore cyst germination of three Phytophthora spp. Plant Dis. 84:454458.

22. Reuveni, M. 2003. Activity of the new fungicide benthiavalicarb against Plasmopara viticola and its efficacy in controlling downy mildew in grapevines. Eur. J. Plant Pathol. 109:243-251.

23. Rubin, E., and Cohen, Y. 2006. An improved method for infecting tomato leaves or seedlings with oospores of Phytophthora infestans used to investigate F1 progeny. Plant Dis. 90:741-749.

24. Rubin, E., Hadad, T., Gisi, U., and Cohen, Y. 2006. Mutagenesis of Phytophthora infestans for resistance against dimethomorph and mefenoxam. (Abstr.) Phytoparasitica 34:305-306.

25. Rubin, E. A., Gotlieb, D., Galperin, M., Gisi, U., and Cohen, Y. 2007. Failure to induce resistance against mandipropamid in Phytophthora infestans. (Abstr.) Phytoparasitica 35:196

26. Stein, J. M., and Kirk, W. W. 2004. The generation and quantification of resistance to dimethomorph in Phytophthora infestans. Plant Dis. 88:930-934.

27. Yuan, S. K., Liu, X. L., Si, N. G., Dong, J., Gu, B. G., and Jiang, H. 2006. Sensitivity of Phytophthora infestans to flumorph: in vitro determination of baseline sensitivity and the risk of resistance. Plant Pathol. 55:258-263.

28. Zhu, S. S., Liu, X. L., Liu, P. F., Li, Y., Li, J. Q., Wang, H. M., Yuan, S. K., and Si, N. G. 2007. Flumorph is a novel fungicide that disrupts microfilament organization in Phytophthora melonis. Phytopathology 97:643649.

29. Ziogas, B. N., Markoglou, A. N., Theodosiou, D. I., Anagnostou, A., and Boutopoulou, S. 2006. A high multi-drug resistance to chemically unrelated oomycete fungicides in Phytophthora infestans. Eur. J. Plant Pathol. 115:283-292. 